\title{
Mechanisms of action of noradrenaline in the brain
}

\author{
MENAHEM SEGAL \\ The Weizmann Institute of Science, Rehovot, Israel
}

\begin{abstract}
Noradrenaline(NA)-containing fibers originate in the nucleus locus coeruleus (LC) and innervate wide cortical and subcortical structures. Extensive evidence indicates that NA of LC origin can suppress apparent spontaneous activity of many neurons in the brain, yet increase their reactivity to certain stimuli. The type of stimuli as well as the nature of the enhancement are region specific. The observed effects of activation of LC are a product of an effect on remote afferents, on local interneurons, and on the projection neuron that is being monitored. The cellular mechanisms underlying these actions have been studied with intracellular recording techniques in the slice preparation. NA hyperpolarizes hippocampal neurons probably by activating an electrogenic $\mathrm{Na}-\mathrm{K}$ pump. It enhances reactivity to afferent stimulation probably by acting presynaptically and also by suppressing a postsynaptic hyperpolarizing Ca-dependent $\mathrm{K}$ current. This results in enhancement of the signal-to-noise ratio of the monitored system and in a more efficient response to significant environmental simuli.
\end{abstract}

The noradrenaline (NA) innervation of the brain has been studied extensively over the past decade (Foote, Bloom, \& Aston-Jones, 1983). Early views suggested that the nucleus locus coeruleus (LC) had a widespread, diffuse, slow-conducting projection system with little anatomical or functional specificity. These views were supported by the observations that: (1) the LC projects into nearly every brain structure in a rather diffuse manner (Lindvall \& Björklund, 1974; Pickel, Segal \& Bloom, 1974); (2) LC fibers are small, unmyelinated, and slow conducting, and therefore unlikely to convey specific information; (3) action potential discharge rates of LC neurons vary considerably, with sleep wakefulness cycles being nearly silent in sleep, especially in REM sleep (Aston-Jones \& Bloom, 1981a; Hobson, McCarley, \& Wyzinski, 1975); and (4) iontophoretic application of NA produces, in nearly every brain area studied, a long-lasting beta receptor-mediated suppression of spontaneous action potential discharges (Foote et al., 1983). It is now becoming apparent that these views are rather simplistic and that LC functions might be more specific and complex. Extensive anatomical studies indicate that LC innervation of the forebrain is quite heterogeneous, with some structures being heavily invested with NA fibers and others being relatively devoid of them. Furthermore, within a given structure, for example, the neocortex, there is a distinct laminar distribution of NA fibers and receptors (Morrison, Foote, Molliver, Bloom, \& Lidov, 1982). The

This article is based on a roundtable discussion held at the European Brain and Behavior Society Workshop on Brain Plasticity, Learning and Memory held August 30-September 1, 1984, in Strasbourg, France.

The author's mailing address is: Center for Neuroscience, The Weizmann Institute of Science, Rehovot, Israel. physiological properties of rat LC neurons, characterized in a recent series of studies (Aston-Jones \& Bloom, 1981a), also indicate that LC might be involved in more complex functions in certain brain regions. First, LC neurons respond vigorously to sensory stimuli with a shortlatency excitation (Aston-Jones \& Bloom, 1981b). Second, they demonstrate a rapid habituation to nonsignificant stimuli (Jones, Segal, Foote, \& Bloom, 1979). Third, their axonal conduction velocity is not as slow as originally thought, especially in large mammals (e.g., monkeys) (Segal, Foote, \& Aston-Jones, 1983). Finally, there are strong indications that NA of LC origin plays an important role in developmental plasticity (Kasamatsu \& Pettigrew, 1976). Those observations militate against the view that the LC is involved only in a global function associated with sleep-wakefulness cycles.

The search for the mechanisms of action of NA in the brain has to be performed at three different levels of analysis. At the behavioral level, effects of stimulation or lesion of the LC on behavioral functions are assessed. At the physiological level, the effects of stimulation of LC or iontophoretic application of NA on firing properties of single cells or cell assemblies can be assessed. The third level of analysis can best be performed in an isolated, in vitro preparation. There, effects of NA on single species of membrane currents can be analyzed. A meaningful theory of action of NA should integrate results from the three levels of analysis. Results from one level of analysis can be of heuristic value for the other levels, and this could reduce the number of possible mechanisms to study at the other levels. Because a number of authors have addressed the behavioral significance of LC activation elsewhere (Mason, 1981; Sara, 1985), the following review will concentrate on the physiological and biophysical analysis. 


\section{EXTRACELLULAR RECORDINGS: PHYSIOLOGICAL MECHANISMS}

Most of the early studies that employed iontophoresis of NA onto neurons throughout the brain reported that it caused suppression of spontaneous action potential discharges. According to Segal and Bloom (1974), this depressant action was mediated by activation of a beta receptor. Excitatory actions were found in cortex, geniculate nucleus (Rogawski \& Aghajanian, 1980), spinal cord (Marshall, 1983), and facial nucleus (Vandermaelen \& Aghajanian, 1982). As suggested by Waterhouse, Moises, \& Woodward (1981), the excitatory effects were likely to be mediated by activation of a postsynaptic alpha receptor. The effects of electrical stimulation of the nucleus locus coeruleus (LC) on spontaneous activity of postsynaptic neurons were tested in the cerebellum (Hoffer, Siggins, Oliver, \& Bloom, 1973), hippocampus (Segal \& Bloom, 1976), neocortex (Phillis \& Kostepoulus, 1977), septum (Segal, 1976), spinal cord (Sasa, Minekiyo, Ikeda, \& Takaori, 1974), thalamus (Rivner \& Sutin, 1981), and other nuclei (see Foote et al., 1983, for a review).

The response to the stimulation involves, in general, a suppression of spontaneous action potential discharge rate. This effect can usually be observed when a train of stimuli is used. It is slow in onset, with a latency as long as several hundreds of milliseconds, and has a long duration of up to several seconds (Figure 1). This type of response is quite different from the typical inhibitory postsynaptic potential (IPSP) seen in other, classical inhibi-
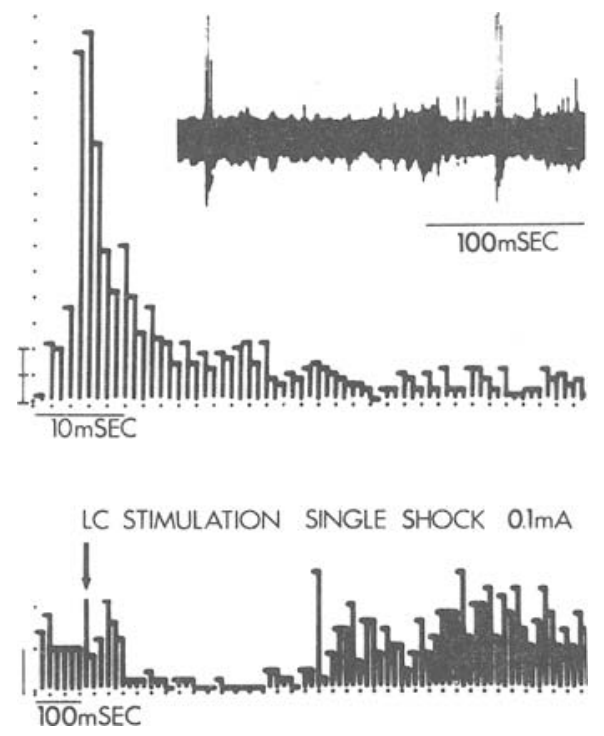

Figure 1. Activity of a single hippocampal neuron recorded in the awake, freely moving rat. The cell has a characteristic bursting firing pattern (top record) with a 5-6-msec interspike interval within a burst and long interburst intervals. This is summarized in an interspike interval histogram (middle record). Single shock stimulation of the LC (0.2-msec pulse) produces a long-latency $(80 \mathrm{msec})$ and -duration ( $500 \mathrm{msec}$ ) suppression of spontaneous activity of the cell. Poststimulus histogram sums 100 responses (bottom). (From Segal \& Bloom, 1976.) tory connections. The inhibitory response can be potentiated by a NA uptake inhibitor and blocked by a beta adrenergic antagonist (Segal \& Bloom, 1976). Also, it is absent in brains treated with drugs that interfere with noradrenergic transmission.

Although suppression of spontaneous activity is the most commonly observed action of NA of LC origin, it is by no means the only action and perhaps not the main one. In the auditory cortex of the squirrel monkey, the effect of NA in reducing responses to auditory stimuli is less than its effect on spontaneous activity (Foote, Freedman, \& Oliver, 1975). In the awake hippocampus, LC stimulation can facilitate excitatory response to a significant, conditioned stimulus (Segal \& Bloom, 1976). In the cerebellum (Freedman, Hoffer, Woodward, \& Puro, 1977), a low dose of NA or LC stimulation can potentiate reactivity of purkinje cells to iontophoretic administration of GABA and other amino acids. A similar effect is seen in the somatosensory region of the neocortex (Waterhouse et al., 1981; Woodward, Moises, Waterhouse, Hoffer, \& Freedman, 1979). The apparent dual action of NA has been studied extensively in the rat visual cortex (Madar, 1983; Madar \& Segal, 1980). The advantages of using this system in the study of functionally identifiable neurons are that the response patterns to visual stimuli can be quantified and that any changes in these can be easily detected. Stimulation of LC or iontophoretic application of NA suppressed spontaneous activity of complex cells via activation of beta-1 adrenoreceptors. Stimulation of LC enhanced specific activity of both simple and complex cells. This specific activity was evoked by a visual stimulus, a bar light, moving in a certain direction and orientation. The response pattern to the stimulus was modified during stimulation; the duration of the visual response was shortened, but its magnitude was, in many cases, enhanced. This enhancement was more evident when the visual stimulation was applied in the preferred direction and orientation (Figure 2). The enhanced reactivity was observed in both simple and complex cells.

Several experiments were performed to analyze the mechanisms underlying the effects of LC stimulation. First, an attempt was made to duplicate these effects by applying NA iontophoretically. NA suppressed spontaneous activity of the recorded neurons. The effects of NA on evoked visual responses ranged from a proportional suppression (much like the effect on background activity) to an absolute enhancement. The quality and magnitude of the effect was dependent, to a large extent, on the amount of NA released; the higher NA concentrations resulted in suppression of both background and evoked activity (Madar, 1983; Madar \& Segal, 1980).

The relative contribution of the NA innervation of the lateral geniculate nucleus (LGN) to the observed effects of LC stimulation in the cortex was assessed by specific lesions of the noradrenergic innervation of the neocortex. This was achieved by transverse knife cuts made at the level of somatosensory cortex. These cuts disrupt the caudally traveling cortical noradrenergic fibers which 

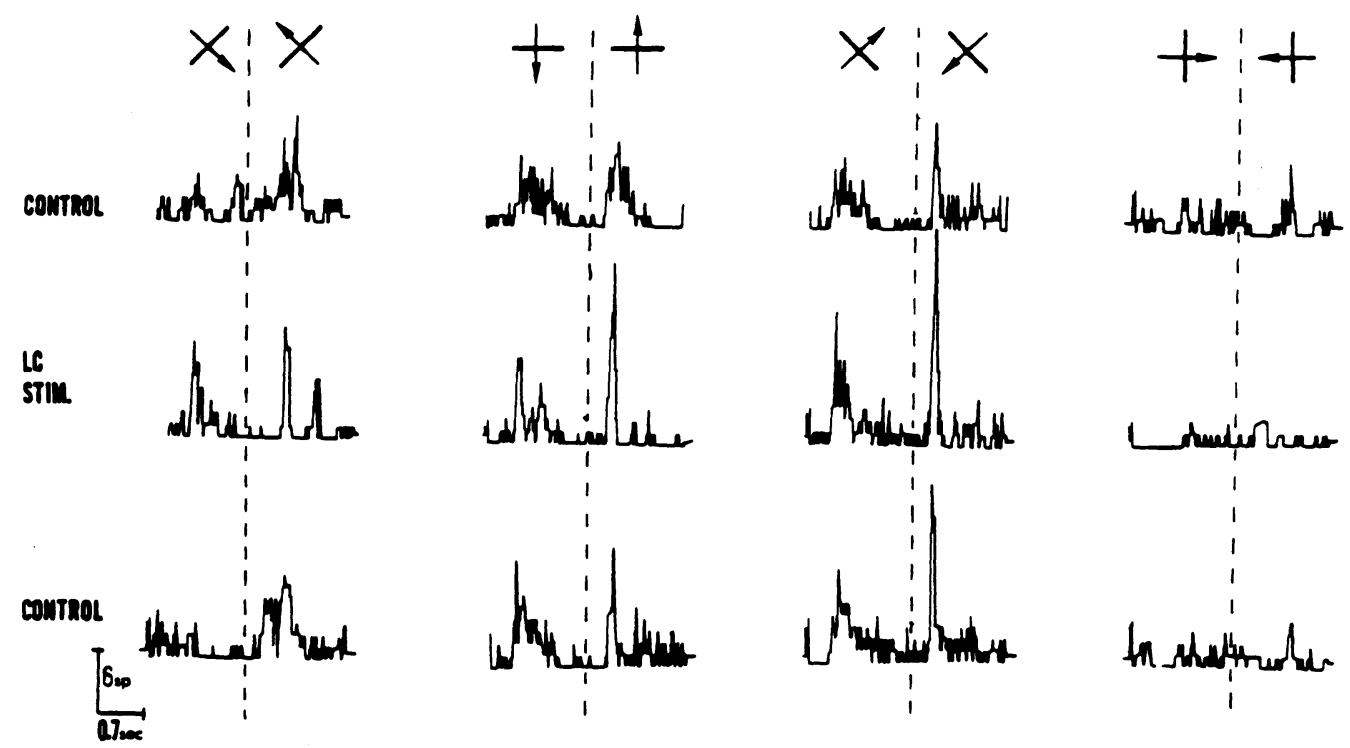

Figure 2. An orientation-selective LC potentiation of the response of a complex cell in rat visual cortex. Orientation response histograms were constructed from the response to a light bar $\left(14^{\circ}\right)$ moved across the entire receptive field in four different orientations, indicated by arrows above each set of histograms. The effect of LC stimulation on the response to each orientation was tested separately. The facilitatory action was more pronounced as the visual stimulus was more optimally oriented, whereas the suppressive action was strengthened as the visual stimulus was oriented away from the optimal orientation axis. Note the better separation of the responsive regions in the receptive field that was produced by LC stimulation. Calibration: horizontal, 0.7 sec; vertical, 6 counts per bin. (From Madar, 1983.)

reach the visual cortex. Under these conditions, LC stimulation could still cause a facilitation of the visual response, but this was now less specific. Also, the effects of LC stimulation on background activity were no longer seen. It therefore appears that NA of LC origin can modulate visual reactivity at two levels; in the LGN, NA facilitates visual responses nonselectively by activating alpha adrenoreceptors, as seen elsewhere (Rogawski \& Aghajanian, 1980). The effects of NA in the visual cortex involves both a beta adrenergic suppression of spontaneous background activity and a selective enhancement of reactivity to some aspects of visual stimuli. The concomitant action of LC in the LGN and visual cortex will bring about the observed increase in signal(visual responses)-to-noise (background activity) ratio. Under ideal conditions, there will be a concurrent increase in responsiveness and a decrease in noise. This will result in sharpened visual functions, that is, a sharper and more defined receptive field and a narrower tuning curve for orientation and direction selectivity. These improved visual responses will result in more refined visual acuity and perhaps enhanced behavioral-visual functions. Such conditions will be observed when the organism attends to its environment, and indeed behavioral studies have indicated that visual cortex neurons of an aroused cat have sharper receptive fields (Livingstone \& Hubel, 1981).

It is conceivable that LC acts simultaneously at different levels of information processing in other neural systems as well. In the hippocampal circuit, the medial septum and dentate gyrus are heavily invested with NA-containing fibers. Although the relative contribution of each relay station to the observed effects of NA of LC origin in the hippocampus proper is not totally understood, it is clear that evoked responses in the hippocampus are markedly affected by behavioral states and by stimuli known to activate the LC (Segal, 1977). These effects cannot be explained by an action of NA at a single site in the hippocampal circuit.

Evidently, the recorded action of NA of LC origin at a given site is a product of NA action at three levels: the remote action (e.g., an effect on LGN when recording is done in the neocortex), the direct postsynaptic action (i.e., to depolarize or hyperpolarize the recorded neuron), and the action on the local circuit. The latter site of action is more difficult to assess in the intact brain, but there is considerable evidence to suggest that NA may affect release of GABA or glutamate and thus determine indirectly the level of excitability of the recorded neuron (Jahr \& Nicoll, 1982). A search for the ionic mechanism(s) of action of NA in a simplified, in vitro preparation should consider these possibilities.

\section{INTRACELLULAR RECORDINGS: IONIC MECHANISMS}

Early intracellular studies done in the intact brain have indicated that NA can hyperpolarize recorded neurons by 5-10 mV. This hyperpolarization can account for the suppression of spontaneous action potential discharge rate. In an extensive study with rat cerebellar purkinje cells (Hoffer et al., 1973), it was shown that a similar hyperpolarization can be evoked by electrical stimulation of the LC. The hyperpolarizing response was associated with an increase or no change in input resistance. It was sug- 
gested that NA acts by increasing intracellular cyclic adenosine monophosphate (CAMP), which acts as a second messenger to phosphorylate some membrane proteins and cause the observed conductance changes. There is, indeed, a large body of evidence to suggest that NA causes an increase in CAMP (Segal, Greenberger, \& Hofstein, 1981). The relation of this to the observed conductance changes remains unclear.

An attempt to assess the conductance mechanism underlying the observed hyperpolarizing response was made by systematically changing the membrane potential and monitoring the potential changes produced by NA. Studies in spinal cord neurons indicate that NA may produce a reduction in both NA and $\mathrm{K}$ conductance, as the reversal potential was about $-15 \mathrm{mV}$ and did not involve a change in Cl conductance (Marshall, 1983; Marshall \& Engberg, 1979). The observed increase in input resistance also seen in hippocampal neurons (Herrling, 1981) would accordingly enhance reactivity to afferent stimulation; an injected synaptic current would produce a larger potential change when input resistance was higher.

Another possible mechanism activated by NA involves the Na-K electrogenic pump. Evidence in favor of such a mechanism has been accumulated in both in vivo and in vitro preparations (Phillis \& Wu, 1981). Ouabain, a Na-K ATPase inhibitor, antagonized responses to NA in vivo (Phillis \& Wu, 1981). Stimulation of Na-K ATPase by NA has been reported in several tissues, including rat brain synaptosomal membranes. This activation in cortical synaptosomes could be blocked by either alpha or beta adrenergic antagonists (Phillis \& Wu, 1981).

The in vitro hippocampal slice preparation has been used for the analysis of the action of NA (Segal, 1981). Topical application of NA produces a transient, 3-5-mV hyperpolarizing response associated with little conductance change. This response could not be reversed either by somatic hyperpolarization or depolarization but was reduced markedly at a low temperature. Also, the response was blocked by ouabain (Figure 3 ). These experiments further support the contention that NA activates a $\mathrm{Na}-\mathrm{K}$ pump that hyperpolarizes the recorded neuron and causes suppression of action potential discharge rate. This mechanism does not explain the increased reactivity to afferent stimulation observed in the hippocampus and elsewhere (Segal \& Bloom, 1976). The hippocampal-slice preparation is also amenable to the analysis of the mechanism of increased reactivity to afferent stimulation: activation of a beta adrenergic receptor is responsible for a severalfold increase in population spike response to stimulation of the excitatory schaffer collateral system (Mueller, Hoffer, \& Dunwiddie, 1981) (Figure 4). Activation of an alpha adrenoreceptor can suppress this population spike response. It has been suggested that the increase in action potential discharge underlying the population spike is not preceded by an increase in the excitatory postsynaptic potential (EPSP) and can thus be considered to take place at the spike initiating zone (Mueller et al., 1981). Using intracellular recording, I have tested the possible effects of NA on reactivity to iontophoretic administration of glutamate (Segal, 1982). NA, acting via a beta receptor, caused a marked increase in the depolarizing response to glutamate (Figure 5) and subsequently increased the number of action potentials discharged during the depolarization. A possible mechanism underlying this effect was described recently by two independent groups (Haas \& Konnerth, 1983; Madison \& Nicoll, 1982). They suggested that NA, acting on a beta receptor, blocked the long afterhyperpolarization (AHP) that

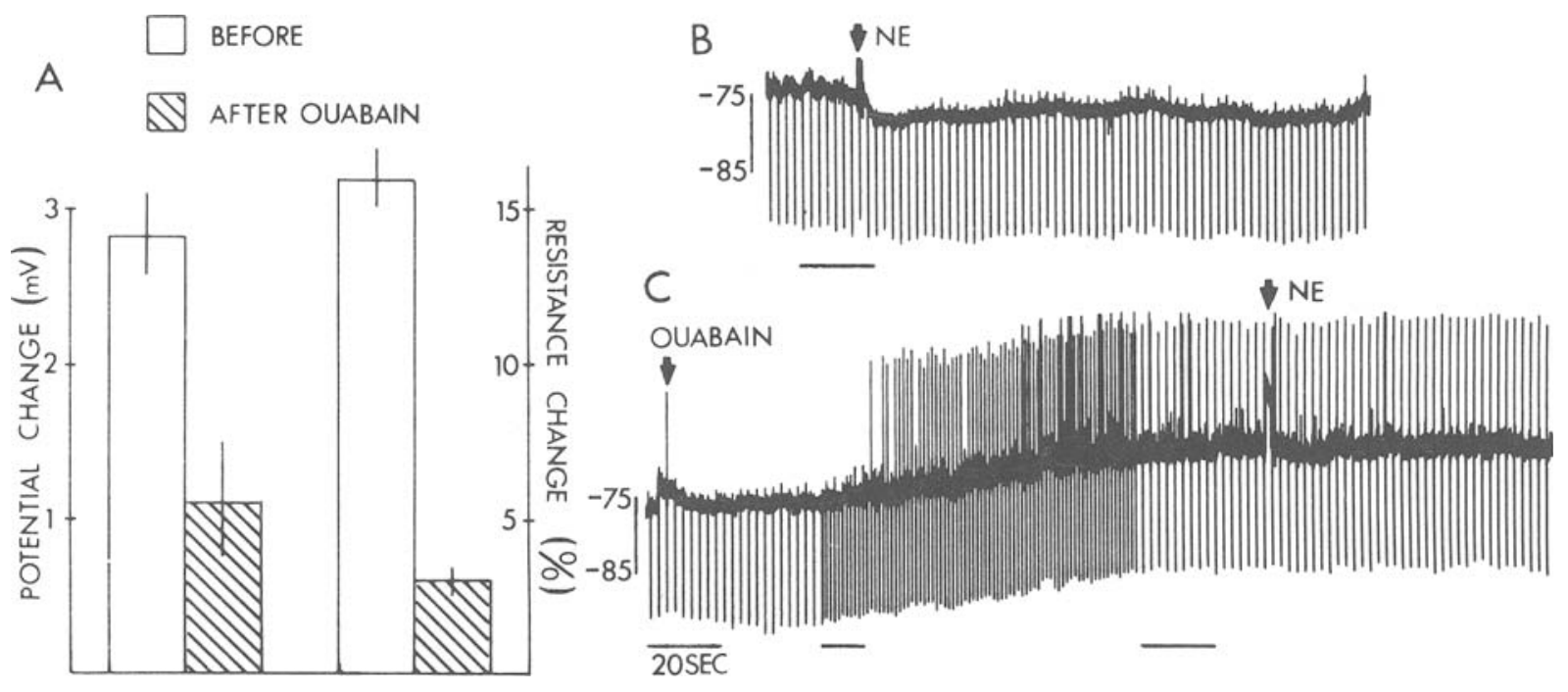

Figure 3. Effects of ouabain on intracellular responses to NA in the rat hippocampal slice. (A) Averages of potential (left) and resistance (right) changes produced by topical application of a microdrop containing $0.5 \mathrm{mM}$ NA before and after topical application of ouabain. (B) Responses of a cell to NA. (C) A drop application of ouabain caused a gradual depolarization associated with the appearance of spike discharges. Same cell as in (B). Application of NA following the application of ouabain did not produce any change in resting potential or input resistance. Note the change in the time base in $\mathrm{C}$ indicated by a shorter horizontal bar and the return to the preceding time base before application of NA. (From Segal, 1981.) 


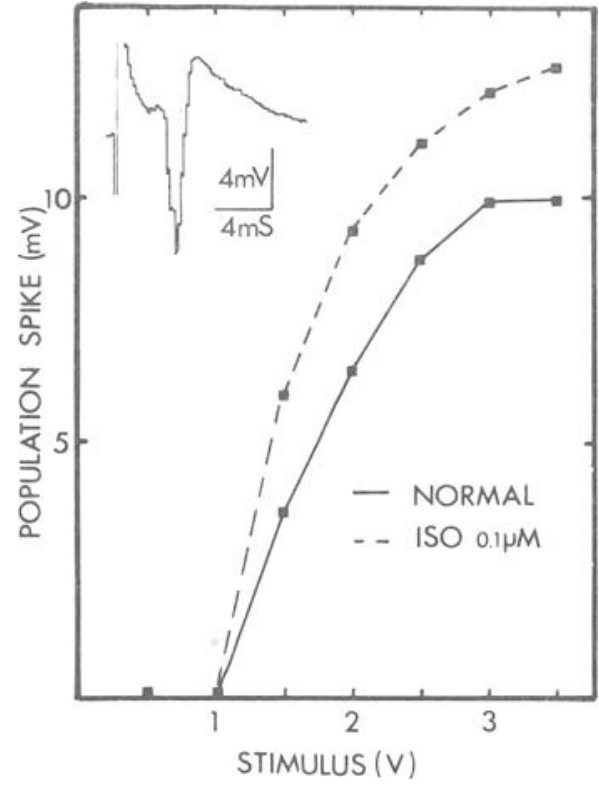

Figure 4. Isoproterenol (ISO) potentiates population spike response to stimulation of the Schaffer collateral system in the hippocampal slice. Responses were digitized and averaged for each stimulus strength before and during superfusion of the slice with isoproterenol.

follows a burst discharge (Figure 6). This AHP reflects activation of a CA-dependent $\mathrm{K}$ current $\left[\mathrm{I}_{\mathrm{K}(\mathrm{CA})}\right]$ that is triggered by an increase in intracellular $\mathrm{CA}^{2+}$ in a voltagedependent manner and functions to repolarize the cell. The effect of NA on AHP is different from the suppressive effect of NA on $\mathrm{I}_{\mathrm{Ca}}$ observed in sympathetic ganglion (Galvan \& Adams, 1982; Horn \& McAfee, 1979), inasmuch as the effect of NA on the $\mathrm{Ca}^{2+}$ spike in the hip- pocampus is only minimal in comparison with its effects on the AHP. Activation of $\mathrm{I}_{\mathrm{K}(\mathrm{Ca})}$ may underlie the accommodation seen in a train of action potential discharge to a long depolarizing current pulse. Indeed, NA blocks this accommodation (Madison \& Nicoll, 1982). The functional significance of this blocked accommodation is not entirely clear, but since glutamate may activate $\mathrm{I}_{\mathrm{K}(\mathrm{Ca})}$, the blockade of this conductance by NA may result in the larger depolarizing response observed in my experiments ( $\mathrm{Se}$ gal, 1982). This blockade of $I_{K(C a)}$ is probably not responsible for the large increase in population spike response to orthodromic stimulation (Mueller et al., 1981) because activation of $\mathrm{I}_{\mathrm{K}(\mathrm{Ca})}$ is a slower process than the initial action potential discharge underlying the population spike.

Two other mechanisms of action of NA have been suggested recently. In the guinea pig hippocampal slice NA was found to reduce an inward rectifying process that is expressed as a nonsymmetrical change in input resistance (Langmoen, Segal, \& Andersen, 1981). It has also been suggested (Jahr \& Nicoll, 1982) that, in the olfactory bulb, NA attenuates inhibitory feedback connections, which will, indirectly, enhance reactivity of the neurons to afferent stimulation. It is conceivable, then, that NA has at least three and perhaps more modes of action. The relevance of any of these to the physiological actions of NA seen in the intact brain is still not clear. There are several differences between the responses to NA in the intact brain and the slice. The alpha facilitatory and beta depressive actions seen in vivo are not maintained in vitro, where the facilitatory action is mediated by a beta adrenoreceptor. Also, stimulation of LC in the awake rat can produce a pronounced and long-lasting suppression of spontaneous activity. Such an effect can be seen only occasionally in the slice preparation. Needless to say,

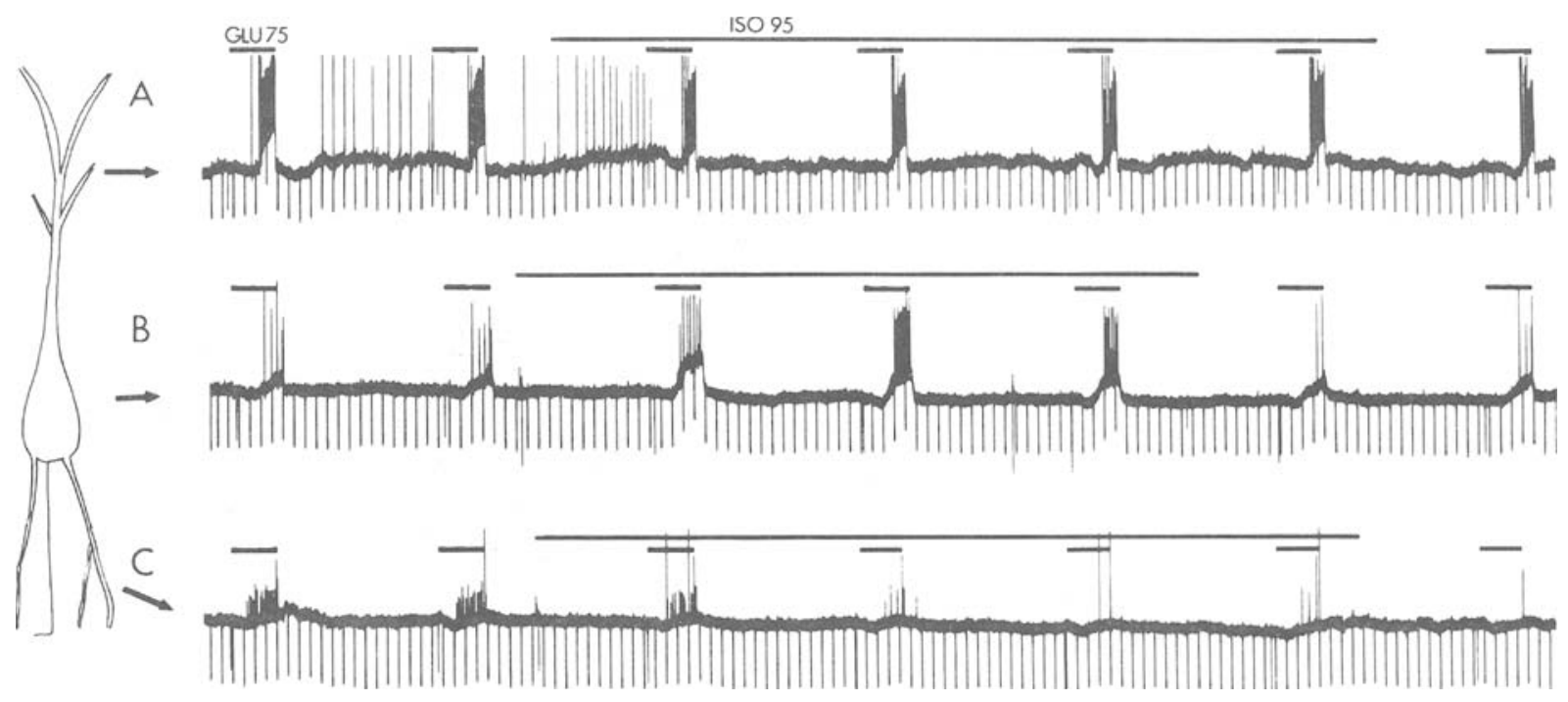

Figure 5. Isoproterenol (ISO) potentiates intracellular responses of a CA1 hippocampal neuron to iontophoretic application of glutamate (GLU). Apical dendritic application of ISO produced a slight hyperpolarization and cessation of spontaneous spike discharges, yet it did not affect the responsiveness to GLU. Basal dendritic application (C) of ISO produced a suppression of reactivity to GLU. Somatic application (B) produced a 2- to 3-mV hyperpolarization and a marked increase in reactivity to GLU. (From Segal, 1982.) 

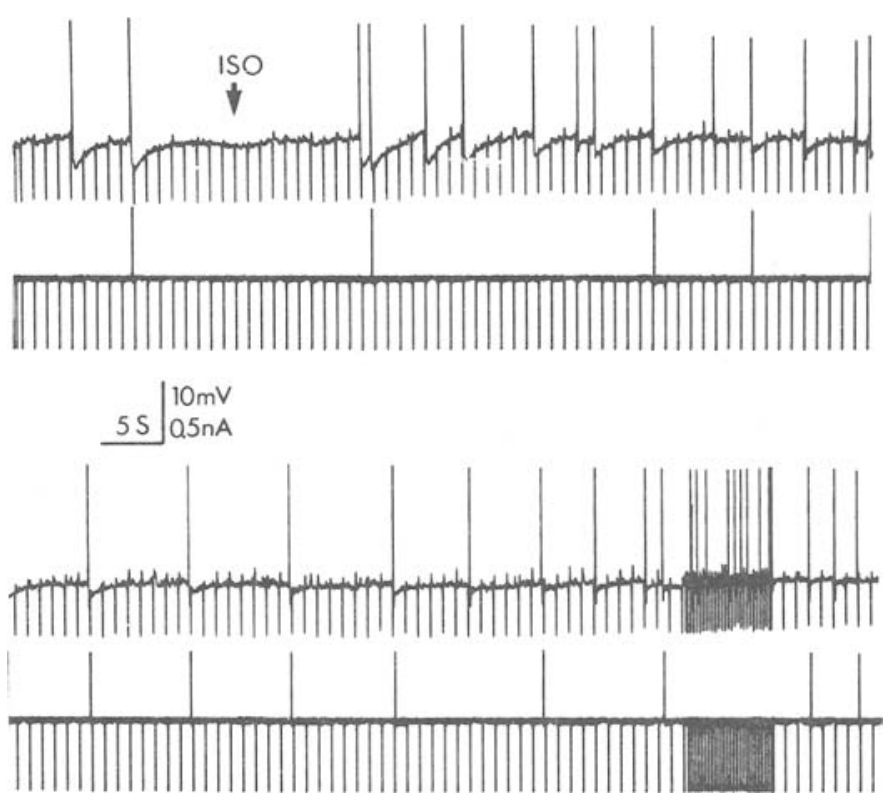

Figure 6. Isoproterenol (ISO) suppresses spontaneous and depolarizationevoked burst after hyperpolarization (AHP) in a CA1 pyramidal cell recorded in a slice. The two pairs of traces (top voltage, bottom current) are continuous. A spontaneous burst (top left) is followed by a slow ( $2 \mathrm{sec})$, large $(5 \mathrm{mV})$ AHP. A depolarizing pulse (upward deflection top left) produces the same AHP. Topical application of ISO $(0.05 \mathrm{mM})$ in a microdrop produces little potential and resistance change, yet facilitates transiently burst discharges. The AHP gradually disappears and does not recover 5 min after ISO application. Hyperpolarizing current pulses are applied at a rate of $1 \mathrm{~Hz}$. Note the change in time base at bottom right.

there are a number of differences between the physiological and biochemical condition of the slice and the brain; the slice contains about half as much ATP as the hippocampus in situ and there is good reason to believe that the slice is somewhat hypoxic. This hypoxia may lead to accumulation of $\mathrm{Ca}$ inside cells and enhance, artificially, detection of $\mathrm{I}_{\mathrm{K}(\mathrm{Ca})}$ and modulations of this conductance mechanism. These conditions may not favor activation of an energy-dependent process by NA (e.g., the slow hyperpolarization) and, instead, may amplify detection of other mechanisms acted upon by NA.

Altogether, it is quite evident that NA acts to suppress spontaneous activity and to enhance evoked responses using one or more of the mechanisms suggested here. The action of NA on the local circuit and its interaction with linked brain regions may bring about the observed behavioral consequences of activation of LC.

\section{REFERENCES}

Aston-Jones, G., \& Bloom, F. E. (1981a). Activity of norepinephrinecontaining locus coeruleus neurons in behaving rats anticipates fluctuations in the sleep waking cycle. Journal of Neuroscience, 1, 876-886.

Aston-Jones, G., \& Bloom, F. E. (1981b). Norepinephrine-containing locus coeruleus neurons in behaving rats exhibit pronounced responses to non-noxious environmental stimuli. Journal of Neuroscience, 1 , 887-900.
Foote, S. L., Bloom, F. E., \& Aston-Jones, G. (1983). Nucleus locus coeruleus: New evidence of anatomical and physiological specificity. Physiological Reviews, 63, 844-914.

Foote, S. L., Freedman, R., \& Oliver, A. P. (1975). Effects of putative neurotransmitters on neuronal activity in monkey auditory cortex. Brain Research, 86, 229-242.

Freedman, R., Hoffer, B. J., Woodward, D. J., \& Puro, R. D. (1977). Interaction of norepinephrine with cerebellar activity evoked by mossy and climbing fibers. Experimental Neurology, 55, 269-288.

Galvan, M., \& Adams, P. R. (1982). Control of calcium current in rat sympathetic neurons by norepinephrine. Brain Research, 244, 135-144.

HaAs, H. L., \& KonNerth, A. (1983). Histamine and noradrenaline decrease calcium-activated potassium conductance in hippocampal pyramidal cells. Nature, 302, 432-434.

HerRLing, P. L. (1981). The membrane potential of cat hippocampal neurons recorded in vivo displays four different reaction mechanisms to iontophoretically applied transmitter agonists. Brain Research, 212, 331-343.

Hobson, J. A., McCarley, R. W., \& Wyzinski, P. W. (1975). Sleep cycle oscillation: Reciprocal discharge by two brainstem neural groups. Science, 198, 55-58.

Hoffer, B. J., Siggins, G. R., Oliver, A. P., \& Bloom, F. E. (1973). Activation of the pathway from locus coeruleus to rat cerebellar purkinje neurons: Pharmacological evidence of noradrenergic central inhibition. Journal of Pharmacology \& Experimental Therapeutics, 184, 553-569.

HoRn. J. P.. \& MCAfEe. D. A. (1979). Norepinephrine inhibits calcium dependent potentials in rat sympathetic neurons. Science, 204, 1233-1235.

Jahr, C. E., \& Nicoll, R. A. (1982). Noradrenergic modulation of dendrodendritic inhibition in the olfactory bulb. Nature, 297, 227-229. 
Jones, G., Segal, M., Foote, S., \& Bloom, F. E. (1979). Locus coeruleus neurons in freely moving rats exhibit pronounced alterations of discharge rates during sensory stimulation. In E. Usdin, E. Copin, \& J. Barchas (Eds.), Catecholamines, basic and clinical frontiers. New York: Pergamon Press.

Kasamatsu, T., \& Pettigrew, J. D. (1976). Depletion of brain catecholamines: Failure of ocular dominance shift after monocular occlusion in kittens. Science, 194, 206-209.

Langmoen, I. A., Segal, M., \& Andersen, P. (1981). Mechanisms of norepinephrine actions on hippocampal pyramidal cells in vitro. Brain Research, 208, 349-362.

LINDVALL, O., \& BJöRKLUND, A. (1974). The organization of the ascending catecholamine neuron systems in the rat brain as revealed by the glyoxylic acid fluorescence method. Acta Physiologica Scandinavica Supplement, 412, 1-48.

Livingstone, M. S., \& HUBEl, D. H. (1981). Effects of sleep and arousal on the processing of visual information in the cat. Nature, 291, 554-561.

MADAR, Y. (1983). The noradrenergic action in the visual cortex. Unpublished doctoral dissertation, The Weizmann Institute of Science, Rehovot.

MADAR, Y., \& SEgal, M. (1980). The functional role of the noradrenergic system in the visual cortex. Activation of the noradrenergic pathway, Experimental Brain Research, 41, 14A.

MAdison, D. V., \& Nicoll, R. A. (1982). Noradrenaline blocks accommodation of pyramidal cell discharge in the hippocampus. $\mathrm{Na}$ ture, 299, 636-638.

Marshall, K. C. (1983). Catecholamines and their actions in the spinal cord. In R. A. Davidoff (Ed.), Spinal cord pharmacology. New York: Dekker.

Marshall, K. C., \& Engberg, I. (1979). Reversal potential for noradrenaline-induced hyperpolarization of spinal motoneurons. Science, 205, 422-424.

Mason, S. T. (1981). Noradrenaline in the brain: Progress in theories of behavioral function. Progress in Neurobiology, 16, 263-303.

Morrison, J. H., Foote, S. L., Molliver, M. E., Bloom, F. E., \& Lidov, H. G. D. (1982). Noradrenergic and serotonergic fibers innervate complemenary layers in monkey primary cortex: An immuno-histochemical study. Proceedings of the National Academy of Science (U.S.A.), 79, 2401-2405.

Mueller, A. L., Hoffer, B. J., \& Dunwiddie, T. V. (1981). Noradrenergic responses in rat hippocampus: Evidence for mediation by $\alpha$ and $\beta$ receptors in the in vitro slice. Brain Research, 214, 113-126.

Phillis, J. W., \& Kostepoulus, G. K. (1977). Activation of a noradrenergic pathway from the brain stem to rat cerebral cortex. General Pharmacology, 8, 207-211.

Phillis, J. W., \& WU, P. H. (1981). Catecholamines and the sodium pump in excitable cells. Progress in Neurobiology, 17, 141-184.
Pickel, V., Segal, M., \& Bloom, F. E. (1974). An autoradiographic study of the efferent pathways of the nucleus locus coeruleus. Journal of Comparative Neurology, 155, 15-41.

Rivner, M., \& Sutin, J. (1981). Locus coeruleus modulation of the motor thalamus: Inhibition in nuclei ventralis lateralis and ventralis anterior. Experimental Neurology, 73, 651-673.

Rogawski, M. A., \& Aghajanian, G. K. (1980). Modulation of lateral geniculate neurone excitation by noradrenaline microiontophoresis or locus coeruleus stimulation. Nature, 287, 731-734.

SARA, S. J. (1985). Noradrenergic modulation of selective attention: Its role in memory retrieval. In D. Olton, E. Gamzu, \& S. Corkin (Eds.), Memory dysfunctions: An integration of animal and human research from clinical and preclinical perspectives. Annals of New York Academy of Science, 444, 178-191.

Sasa, M., Minekiyo, K., IKeda, H., \& TaKaori, S. (1974). Noradrenaline-mediated inhibition by locus coeruleus of spinal trigeminal neurons. Brain Research, 80, 443-460.

SEgAL, M. (1976). Brainstem afferents to the rat medial septum. Journal of Physiology, 261, 617-631.

SEgal, M. (1977). The effects of brainstem priming stimulation on interhemispheric hippocampal responses in the awake rat. Experimental Brain Research, 28, 529-541.

SEgAL, M. (1981). The action of norepinephrine in the rat hippocampus: Intracellular studies in the slice preparation. Brain Research, 206, 107-128.

SEGAL, M. (1982). Norepinephrine modulates reactivity of hippocampal cells to chemical stimulation in-vitro. Experimental Neurology, 77, 86-93.

Segal, M., \& Bloom, F. E. (1974). Norepinephrine in the rat hippocampus: Iontophoresis studies. Brain Research, 82, 79-97.

SEgAL, M., \& Bloom, F. E. (1976). The action of norepinephrine in rat hippocampus: IV. The effects of locus coeruleus stimulation on evoked hippocampal unit activity. Brain Research, 107, 513-525.

Segal, M., Foote, S. L., \& Aston-Jones, G. (1983). Physiological properties of ascending locus coeruleus axons in the squirrel monkey. Brain Research, 274, 381-387.

Segal, M., Greenberger, V., \& Hofstein, R. (1981). Cyclic AMP generating systems in rat hippocampal slices. Brain Research, 213, 351-364.

Vandermaelen, C. P., \& Aghajanian, G. K. (1982). Serotonininduced depolarization of rat facial motoneurons in vivo: Comparison with amino acid transmitters. Brain Research, 239, 139-152.

Waterhouse, B. D., Moises, H. C., \& Woodward, D. J. (1981). Alpha-receptor mediated facilitation of somatosensory cortical neuronal responses to excitatory synaptic inputs and iontophoretically applied acetylcholine. Neuropharmacology, 20, 907-920.

Woodward, D. J., Moises, H. C., Waterhouse, B. D., Hoffer, B. J., \& FreEdMAN, R. (1974). Modulatory actions of norepinephrine in the central nervous system. Federation Proceedings, 38, 2109-2116. 